\title{
Performance Evaluation of different Image Filtering Algorithms using Image Quality Assessment
}

\author{
M.Prema Kumar \\ Associate Professor \\ Department of ECE \\ Sri Vishnu Engineering College \\ for Women, Bhimavaram.
}

\author{
P.H.S.Tejo Murthy \\ Assistant Professor \\ Department of EIE, GITAM \\ Visakhapatnam
}

\author{
Dr.P.Rajesh Kumar \\ Associate Professor \\ Department of ECE, AU \\ Visakhapatnam
}

\begin{abstract}
The quality assessment of images is meaningful for most video and images applications. Recently a new metric called Region of Interest structure similarity index (ROISSIM) is proposed for assessing image quality with better reflection to human visual characteristics than traditional image quality assessment methods. In this paper, five different filtering algorithms are compared based on the ability to reconstruct noise affected images using ROISSIM. Experimental results give us the quality assessment of filtering algorithms based on region of interest.
\end{abstract}

\section{Keywords}

Image Quality Assessment, Region of Interest, Image Restoration, Image Processing.

\section{INTRODUCTION}

Order statistics filters exhibit better performance as compared to linear filters [1][2] when restoring images corrupted by impulse noise. Impulse noises are short duration noises which degrade an image. They may occur during image acquisition, due to switching, sensor temperature. They may also occur due to interference in the channel and due to atmospheric disturbances during image transmission. The goal of the filtering action is to cancel noise while preserving the integrity of edge and detail information.

In this paper, five different filtering algorithms are used for the removal of impulse noise in digital images. The performance of filtering methods is evaluated with different noise density using ROI-SSIM image quality assessment method.

This paper is organized as follows: Section II present five different filtering algorithms, Section III presents ROI_SSIM image quality assessment method, Section IV presents Experimental results and finally Section V reports conclusion.

\section{FILTERING ALGORITHMS}

Order-static filters are nonlinear filters whose response is based on the ordering (ranking) the pixels contained in the image area encompassed by the filter, and then replacing the value of the center pixel with the value determined by the ranking result.

The simplest of the algorithms is the Mean Filter: The Mean Filter [3] is a linear filter which uses a mask over each pixel in the signal. Each of the components of the pixels which fall under the mask are averaged together to form a single pixel. This new pixel is then used to replace the pixel in the signal studied. The Mean Filter is poor at maintaining edges within the image. The mean filter is defined as follows

$\operatorname{MEANFILTER}\left(x_{1}, x_{2}, \ldots \ldots x_{N}\right)=\frac{1}{N} \sum_{i=1}^{N} x_{i}$

The Median Filter [4] as the name implies, replaces the value of the pixel by the median of the intensity values in the neighborhood of that pixel defined in (2). The pixel with the median magnitude is used to replace the pixel in the signal studied.

$$
\begin{gathered}
\operatorname{MEDIANFILTER}\left(x_{1}, x_{2}, \ldots \ldots x_{N}\right)= \\
\operatorname{MEDIAN}\left(x_{1}, x_{2}, \ldots \ldots x_{N}\right)
\end{gathered}
$$

The median filter is more robust with respect to the presence of noise.

The Component Median Filter (CMF) [5], defined in (3), also relies on the statistical median concept. In the Simple Median Filter, each point in the signal is converted to a single magnitude. In the Component Median Filter, each scalar component is treated independently. A filter mask is placed over a point in the signal. For each component of each point under the mask, a single median component is determined. These components are then combined to form a new point, which is then used to represent the point in the signal studied.

$C M F\left(x_{1}, x_{2}, \ldots x_{N}\right)=\left\{\begin{array}{l}\operatorname{MEDIAN}\left(x_{1 r}, \ldots \ldots x_{N r}\right) \\ \operatorname{MEDIAN}\left(x_{1 g}, \ldots \ldots x_{N g}\right) \\ \operatorname{MEDIAN}\left(x_{1 b}, \ldots \ldots x_{N b}\right)\end{array}\right.$

In the Vector Median Filter (VMF) [6] for the ordering of the vectors in a particular kernel or mask a suitable distance measure is chosen. The vector pixels in the window are 
ordered on the basis of the sum of the distances between each vector pixel and the other vector pixels in the window. The sum of the distances is arranged in the ascending order and then the same ordering is associated with the vector pixels. The vector pixel with the smallest sum of distances is the vector median pixel. The vector median filter is represented as

$X_{V M F}=$ vectormedian (window)

If $\delta_{\mathrm{i}}$ is the sum of the distances of the $\mathrm{i}^{\text {th }}$ vector pixel with all the other vectors in the kernel, then

$\delta_{i}=\sum_{j=1}^{N} \Delta\left(X_{i}, X_{j}\right)$

where $(1 \leq \mathrm{i} \leq \mathrm{N})$ and $\mathrm{X}_{\mathrm{i}}$ and $\mathrm{X}_{\mathrm{j}}$ are the vectors, $\mathrm{N}=9$.

$\Delta\left(X_{i}, X_{j}\right)$ is the distance measure given by the $L_{1}$ norm or the city block distance which is more suited to non correlated noise. The ordering may be illustrated as

$\delta_{1} \leq \delta_{2} \leq \delta_{3} \leq \ldots, \leq \delta_{9}$

and this implies the same ordering to the corresponding vector pixels i.e.

$X_{(1)} \leq X_{(2)} \leq \ldots, \leq X_{(9)}$

where the subscripts are the ranks. Since the vector pixel with the smallest sum of distances is the vector median pixel, it will correspond to rank 1 of the ordered pixels, i.e, $X_{V M F}=X_{(1)}$

The Spatial Median Filter (SMF) [5] is a uniform smoothing algorithm with the purpose of removing noise and fine points of image data while maintaining edges around larger shapes. The SMF is based on the spatial median quantile function which is a $\mathrm{L}_{1}$ norm metric that measures the difference between two vectors. The spatial depth between a point and a set of points is defined by

$S_{\text {depth }}\left(X, x_{1}, x_{2}, \ldots \ldots x_{N}\right)=1-\frac{1}{N-1}\left\|\sum_{i=1}^{N} \frac{X-x i}{\|\mathrm{X}-\mathrm{x} i\|}\right\|$

Let $\mathrm{r}_{1}, \mathrm{r}_{2}, \ldots . \mathrm{r}_{\mathrm{N}}$ represent $\mathrm{x}_{1}, \mathrm{x}_{2}, \ldots . . \mathrm{x}_{\mathrm{N}}$ in rank order such that $S_{\text {depth }}\left(r_{1}, x_{1}, x_{2}, \ldots \ldots x_{N}\right)$

$\geq S_{\text {depth }}\left(r_{2}, x_{1}, x_{2}, \ldots \ldots x_{N}\right)$

$\geq$

$\geq S_{\text {depth }}\left(r_{N}, x_{1}, x_{2}, \ldots \ldots x_{N}\right)$

and let $\mathrm{r}_{\mathrm{c}}$ represent the center pixel under the mask. Then

$\operatorname{SMF}\left(x_{1}, x_{2}, \ldots \ldots x_{N}\right)=r_{I}$

\section{ROI-SSIM} ASSESSMENT

The Structural Similarity (SSIM) [7] paradigm hypothesized that the comparison between a reference image and a distorted image consists of three factors. They are luminance comparison, contrast comparison and structural comparison.
Given two real valued sequences $x=\left\{x_{1}, \ldots, x_{n}\right\}$ and $y=\left\{y_{1}, \ldots, y_{n}\right\}, \bar{x}$ is the mean of $\mathrm{x}, \quad \sigma_{\mathrm{x}}{ }^{2}$ is variance of $\mathrm{x}$, $\sigma_{y}^{2}$ is variance of $y$ and $\sigma_{x y}$ is the covariance of $x, y$.

$\sigma_{x}^{2}=\frac{1}{n-1} \sum\left(x_{i}-\bar{x}\right)^{2}$

$\sigma_{y}^{2}=\frac{1}{n-1} \sum\left(y_{i}-\bar{y}\right)^{2}$

$\sigma_{x y}=\frac{1}{n-1} \sum_{i=1}^{n}\left(x_{i}-\bar{x}\right)\left(y_{i}-\bar{y}\right)$

Then, SSIM can be computed as

$\operatorname{SSIM}=\frac{\sigma_{x y}}{\sigma_{x} \sigma_{y}} \cdot \frac{2 \overline{x y}}{\bar{x}^{2}+\bar{y}^{2}} \cdot \frac{2 \sigma_{x} \sigma_{y}}{\sigma_{x}^{2}+\sigma_{y}^{2}}$

If the reference image and its distorted image are divided into blocks, then the overall image quality is evaluated by using mean SSIM (MSSIM), given as

$\operatorname{MSSIM}(x, y)=\frac{1}{K} \sum_{j=1}^{K} \operatorname{SSIM}(x, y)$

Instead of calculating similarity of the entire image with the reference image, the ROI-SSIM [8] calculates the similarity between the regions of the two images. A region is an interested part in an image where people tend to focus their sight, while observing an image. The ROI-SSIM works as follows: First, an image is divided into blocks with size of $11 \times 11$ and the structural value $\sigma$, of each block $\sigma$ is calculated. Second the structural value of current block is compared with its neighbors. If the differences are smaller than a threshold, they will be merged up and the structural value of the newly merged block would be updated. Otherwise the block is kept as a region. The whole image is divided into isolated regions which contain number of similar blocks. Set each block in the i-th region with a weight $\mathrm{w}_{\mathrm{i}}$.

$$
w_{i}=\frac{1}{N_{i} X M}(i=1 \text { to } M)
$$

Where $\mathrm{M}$ is the number of divided regions in the reference image, $\mathrm{N}_{\mathrm{i}}$ is the number of blocks in each region.

ROI-SSIM is defined as follows:

$R O I-S S I M=\sum_{j} \sum_{i} w_{i} \operatorname{SSIM}\left(x_{i}, y_{j}\right)$

Where $\mathrm{i}=1$ to $\mathrm{M}$ and $\mathrm{j}=1$ to $\mathrm{N}_{\mathrm{i}}$. 


\section{EXPERIMENTAL RESULTS}

The performance evaluation of different filters using ROISSIM is tested on the true color parrot image with $290 \times 290$ pixels. The impulse noise is added into the image with different noise densities. The noisy image is processed using five different filtering algorithms. The performance of filtered images is evaluated by computing ROI-SSIM between the filtered image and the original image .The experimental results are shown in Figure 1. Table (1) shows the results of MSSIM and ROI-SSIM values of filtered images with different noise densities. The region we are focusing is on the two parrots.

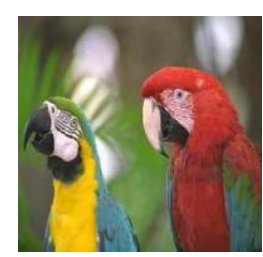

(a)

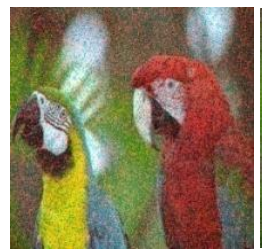

(c)

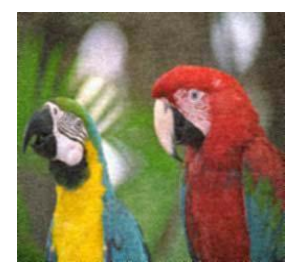

(e)

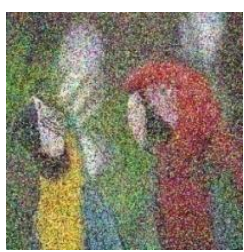

(b)

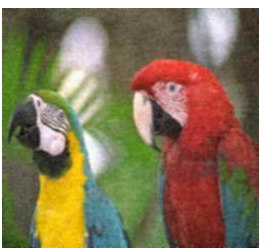

(d)

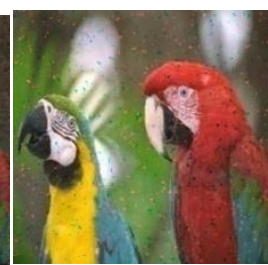

(f)

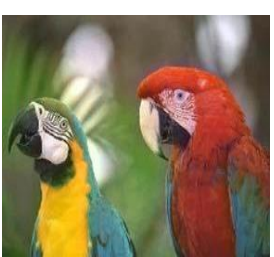

(g)

Figure 1 a) original image, b) impulse noise image corrupted by noise density 0.4 , c) mean filtered image d) median filtered image, d) CMF e) VMF, f) SMF
Table 1: Performance evaluation of filters using ROI_SSIM

\begin{tabular}{|c|c|c|c|c|}
\hline & $\begin{array}{c}\text { Noise } \\
\text { density } \\
0.2 \\
\text { ROI- } \\
\text { SSIM }\end{array}$ & $\begin{array}{c}\text { Noise } \\
\text { Density } \\
0.4 \\
\text { ROI- } \\
\text { SSIM }\end{array}$ & $\begin{array}{c}\text { Noise } \\
\text { Density } \\
0.6 \\
\text { ROI- } \\
\text { SSIM }\end{array}$ & $\begin{array}{c}\text { Noise } \\
\text { Density } \\
0.8 \\
\text { ROI- } \\
\text { SSIM }\end{array}$ \\
\hline Mean & 0.9124 & 0.8187 & 0.7200 & 0.6046 \\
\hline Median & 0.9909 & 0.9619 & 0.8655 & 0.6771 \\
\hline CMF & 0.9896 & 0.9813 & 0.9513 & 0.8430 \\
\hline VMF & 0.9909 & 0.9629 & 0.8643 & 0.6871 \\
\hline SMF & 0.8510 & 0.7381 & 0.6472 & 0.5321 \\
\hline
\end{tabular}

\section{CONCLUSION}

This paper presents the performance evaluation of different filters using ROI_SSIM image quality index. The ROISSIM is better than SSIM for images having distinctive background/foreground. The experimental results also shows that the component median filter works better in removal of impulse noise in digital images than the remaining filters.

\section{REFERENCES}

[1] F.VanderHeijden, Image Based Measurement Systems. NewYork: Wiley, 1994

[2] K. Jain, Fundamentals of Digital Image Processing. Englewood Cliffs, NJ: Prentice-Hall, 1989.

[3] I.Pitas and A.N.Venetsanopoulos, Nonlinear Digital Filters: Principles and Applications. Norwell, MA: Kluwer, 1990.

[4] Rafael C. Gongalez, R.C.Woods, "Digital Speech and Image Processing”, Pearson Education,2009

[5] James C. Church, Yixin Chen, and Stephen V. Rice , "A Spatial Median Filter for Noise Removal in Digital Images“, 2008 IEEE.

[6] S.Indu , Chaveli Ramesh, " A noise fading technique for images highly corrupted with impulse noise", Proceedings of the ICCTA07, IEEE.

[7] Z. Wang and A. C. Bovik, "A universal image quality index," IEEE Signal Processing Letters, vol. 9, pp. 81-84, Mar. 2002.

[8] Yanrui Liu, Han Sun, Yingqi Di and YanZhou, " A New Region of Interest Based Image Quality Assessment Algotithm", 2010 IEEE. 